\title{
An Atypical Presentation of Acute Macular Neuroretinopathy after Non-Ocular Trauma
}

\author{
Robin K. Kuriakose $^{a} \quad$ Eric K. Chin ${ }^{b, c}$ David R.P. Almeida ${ }^{d}$ \\ aLoma Linda University Medical Center, Loma Linda, CA, USA; ${ }^{b}$ Retina Consultants of \\ Southern California, Redlands, CA, USA; ' Loma Linda University Medical Center, Veterans \\ Affair Hospital, Loma Linda, CA, USA; dVitreoRetinal Surgery PA, Minneapolis, MN, USA
}

\section{Keywords}

Acute macular neuroretinopathy · Ischemia · Non-ocular trauma · Paracentral scotoma . Petalloid lesion

\begin{abstract}
Purpose: Acute macular neuroretinopathy $(\mathrm{AMN})$ is a rare clinical entity with an uncertain etiology. We report an atypical case presenting with retinal hemorrhages $(\mathrm{RH})$ and cotton-wool spots (CWS) following non-ocular trauma. Observations: A 49-year-old male presented with an acute onset of a paracentral scotoma in his left eye, immediately following a motor vehicle accident 1 day prior. Fundus findings revealed a unilateral nasal petalloid perifoveal lesion with the tip pointing toward the fovea associated with CWS and RH. Optical coherence tomography demonstrated disruption of the ellipsoid zone. Symptoms and exam findings improved at 2week follow-up without any intervention, consistent with the natural history of the disease process. Conclusion: We report a rare case of AMN following non-ocular trauma with the unique fundus findings of CWS and $\mathrm{RH}$. This presentation supports the role of ischemia in the retinal deep capillary plexus, of which trauma contributed to the pathophysiological process.

Summary: AMN is a rare condition whose pathophysiological process remains speculative. We report an atypical case of $\mathrm{AMN}$, which supports the role of trauma in the pathophysiology of deep retinal capillary plexus compromise.




\section{Introduction}

Acute macular neuroretinopathy (AMN) is a rare condition, first described by Bos and Deutman [1], characterized by one or more dark-reddish oval or wedge-shaped macular lesions pointing toward the fovea with corresponding transient or permanent paracentral scotomas. While the condition is most commonly associated with a recent flu-like illness or with the use of oral contraceptives, 9 cases have been described immediately following trauma [2]. We describe a unique case of AMN following non-ocular trauma associated with unilateral retinal hemorrhages (RH) and cotton-wool spots (CWS), which may advance our understanding of the pathophysiology of this disease.

\section{Case Report}

A 49-year-old healthy male presented with a paracentral temporal scotoma in his left eye after a motor vehicle accident 1 day prior. While the airbag was deployed, the patient did not suffer any direct ocular injury or any other physical injuries. The patient had refractive LASIK OU in 2015 but denied any other past ocular or medical history. Best-corrected visual acuity (BCVA) measured 20/20 OD and 20/25 OS, and intraocular pressures were within normal limits OU. Slit lamp biomicroscopy of the anterior segment was unremarkable OU. Dilated fundus examination OS revealed a nasal petalloid perifoveal lesion with the apex pointing toward the fovea, as well as multiple CWS and RH (Fig. 1).

Optical coherence tomography (OCT) demonstrated hyperreflectivity of the outer plexiform layer and outer nuclear layer with associated disruption of the inner segment/outer segment junction (ellipsoid zone) (Fig. 2). Fluorescein angiography was unremarkable and did not demonstrate leakage. No interventions were performed. At 2-week follow-up, the patient noted subjective improvement in the paracentral scotoma, and examination demonstrated improved paracentral wedge defect, $\mathrm{RH}$, and CWS. At 2-month follow-up, the patient had complete resolution of the clinical retinal findings with near-complete restoration of the subjective paracentral visual complaints.

\section{Discussion}

A rare condition, AMN has been reported 111 times in the literature between 1975 and $2016[2,3]$. Among these, only 9 cases were associated with trauma. The vast majority of those affected with AMN tend to be women, and associated triggers are a nonspecific flu-like illness or fever, oral contraceptive use, and exposure to vasoconstrictive agents such as epinephrine or ephedrine. Our patient presented with a reddish-brown paracentral petalloid lesion with its apex pointing toward the fovea, which is classically seen in patients with AMN. This case describes a rare presentation of AMN following non-ocular trauma secondary to motor vehicle accident. To our knowledge, RH and CWS have never previously been reported in a patient with AMN.

Gillies et al. [4] were the first to report indirect trauma presenting with AMN, proposing that an acute increase in intrathoracic pressure secondary to the accident may cause a sudden rise in intravascular pressure, which could in turn cause a breakdown of the capillary bloodretinal barrier. However, these reports were issued prior to the widespread use and advances of OCT imaging, so the exact location of the retinal defect could not be determined [5]. Using 
OCT imaging, Nentwich et al. [3] in 2013 were the first to show the outer retinal defect in 5 cases of AMN due to non-ocular trauma, supporting the studies by Gillies et al. [4].

The fundoscopic findings of RH and cotton wool spots in the context of non-ocular trauma may also be a presentation of Purtscher retinopathy [6]. Purtscher retinopathy is an occlusive microvasculopathy caused by either cranial trauma or thoracic compression. The initial presentation is vision loss of variable severity, within hours to days of the trauma. The findings of cotton wool spots are found in $93 \%$ of cases, and $\mathrm{RH}$ in $65 \%$ of cases [6]. When considering proposed diagnostic criteria by Miguel et al. [6], this patient would meet 3 of the 5 criteria for diagnosis. Although Purtscher retinopathy alone would not explain this patient's presentation, particularly due to the pathognomonic perifoveal petalloid lesion, it may be a superimposed retinopathy.

Prior studies have suggested ischemic and inflammatory etiologies as a possible cause of AMN. Our findings of cotton wool spots and RH provide further support to the role of ischemia of the deep retinal capillary plexus in AMN. It is important to acknowledge as well that infectious etiologies or other risk factors, such as oral contraceptive usage, can indirectly influence deep retinal capillary perfusion. Additional animal studies, histopathologic correlation, and the use of OCT angiography may help to further support a microvascular etiology for AMN.

\section{Statement of Ethics}

Patient consent was obtained orally, and all patient identifiers were removed from the submission. Full adherence to the Declaration of Helsinki and all Federal and State laws.

\section{Disclosure Statement}

R.K.K.: None. E.K.C.: Citrus Therapeutics (cofounder), Ophthotech, Regeneron, Eyenuk, Opthea, Ohr Pharmaceutical, Tyrogenex Inc., Alimera. D.R.P.A.: Allergan (speaker, consultant, honoraria), Citrus Therapeutics (cofounder), Genentech (speaker, consultant, honoraria), Alcon, Regeneron.

\section{Author Contributions}

All authors attest that they meet the current ICMJE criteria for authorship.

\section{References}

1 Bos PJ, Deutman AF. Acute macular neuroretinopathy. Am J Ophthalmol. 1975 Oct;80(4):573-84.

2 Bhavsar KV, Lin S, Rahimy E, Joseph A, Freund KB, Sarraf D, et al. Acute macular neuroretinopathy: A comprehensive review of the literature. Surv Ophthalmol. 2016 Sep-Oct;61(5):538-65.

3 Nentwich MM, Leys A, Cramer A, Ulbig MW. Traumatic retinopathy presenting as acute macular neuroretinopathy. Br J Ophthalmol. 2013 Oct;97(10):1268-72.

4 Gillies M, Sarks J, Dunlop C, Mitchell P. Traumatic retinopathy resembling acute macular neuroretinopathy. Aust N Z J Ophthalmol. 1997 Aug;25(3 S1):207-10.

5 Grover S, Brar VS, Murthy RK, Chalam KV. Infrared imaging and spectral-domain optical coherence tomography findings correlate with microperimetry in acute macular neuroretinopathy: a case report. J Med Case Reports. 2011 Oct;5(1):536. 


\section{Case Reports in Ophthalmology}

Kuriakose et al.: An Atypical Presentation of Acute Macular Neuroretinopathy after NonOcular Trauma

6 Miguel AI, Henriques F, Azevedo LF, Loureiro AJ, Maberley DA. Systematic review of Purtscher's and Purtscher-like retinopathies. Eye (Lond). 2013 Jan;27(1):1-13.
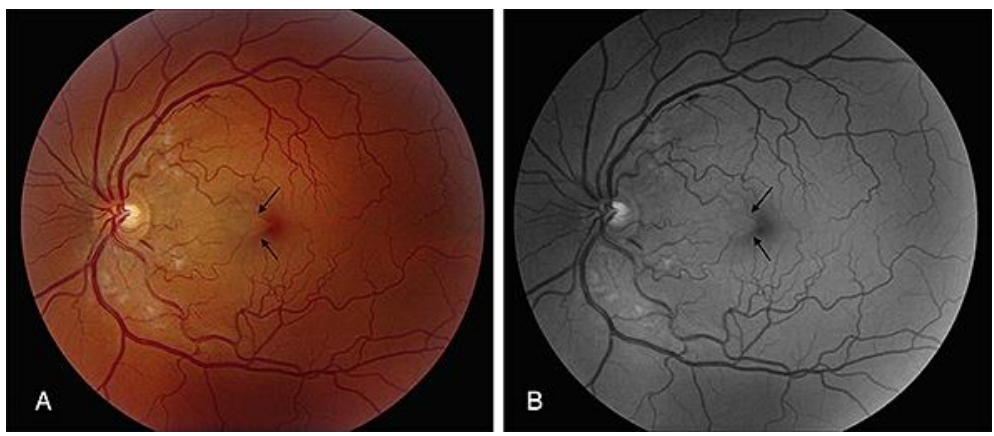

Fig. 1. A, B Color and red-free fundus photo of the left eye demonstrating a brownish-red petalloid lesion on the nasal aspect of the macula (arrows) pointing toward the fovea; several cotton-wool spots along the superotemporal and inferotemporal arcades and multiple retinal hemorrhages.

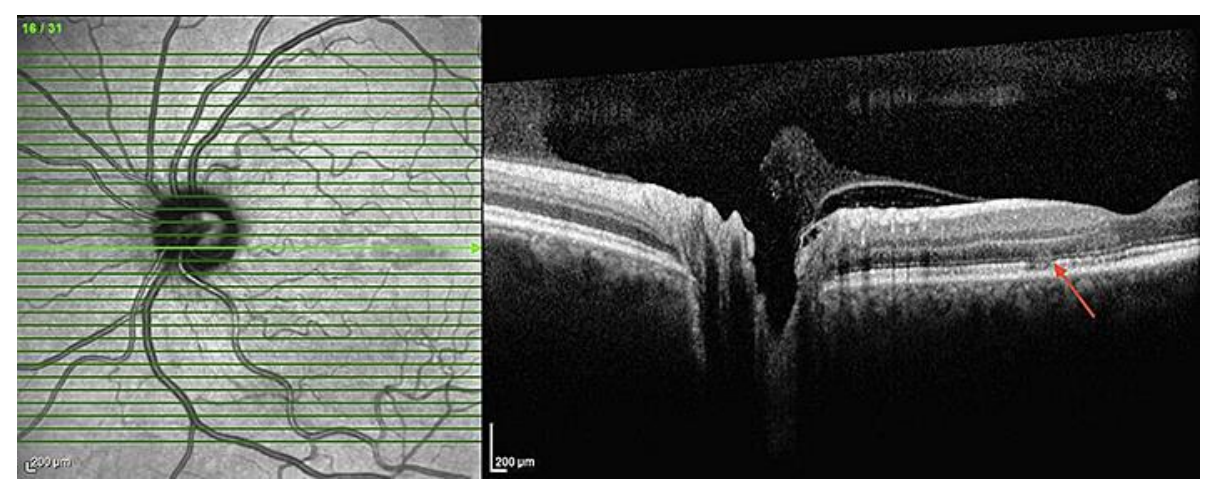

Fig. 2. Infrared fundus photo and OCT of the left eye with hyperreflective plaques at the level of the OPL/ONL junction, with associated disruption of the inner segment/outer segment junction (ellipsoid zone). 